Acta Crystallographica Section F

Structural Biology

and Crystallization

Communications

ISSN 1744-3091

Sachiko Takahashi, ${ }^{\text {a* }}$ Toshiharu

Tsurumura, ${ }^{b}$ Kosuke Aritake, ${ }^{b}$

Naoki Furubayashi, ${ }^{c}$ Masaru

Sato, ${ }^{d}$ Mari Yamanaka, ${ }^{a}$ Erika

Hirota, ${ }^{a}$ Satoshi Sano,

Tomoyuki Kobayashi,d Tetsuo

Tanaka, ${ }^{d}$ Koji Inaka, ${ }^{c}$ Hiroaki

Tanaka $^{a}$ and Yoshihiro Urade ${ }^{b}$

${ }^{\mathbf{a} C o n f o c a l}$ Science Inc., Japan, ${ }^{\mathbf{b}}$ Osaka

Bioscience Institute, Japan, ${ }^{\mathrm{C}}$ Maruwa Foods and Biosciences Inc., Japan, and dapan Aerospace

Exploration Agency, Japan

Correspondence e-mail:

takahashis@confsci.co.jp

Received 26 March 2010

Accepted 1 June 2010

\section{High-quality crystals of human haematopoietic prostaglandin D synthase with novel inhibitors}

Human haematopoietic prostaglandin D synthase (H-PGDS; EC 5.3.99.2) produces prostaglandin $\mathrm{D}_{2}$, an allergic and inflammatory mediator, in mast cells and Th2 cells. H-PGDS has been crystallized with novel inhibitors with halfmaximal inhibitory concentrations $\left(\mathrm{IC}_{50}\right)$ in the low nanomolar range by the counter-diffusion method onboard the Russian Service Module on the International Space Station. The X-ray diffraction of a microgravity-grown crystal of H-PGDS complexed with an inhibitor with an $\mathrm{IC}_{50}$ value of $50 \mathrm{n} M$ extended to $1.1 \AA$ resolution at $100 \mathrm{~K}$ using SPring-8 synchrotron radiation, which is one of the highest resolutions obtained to date for this protein.

\section{Introduction}

Prostaglandin (PG) $\mathrm{D}_{2}$ is a lipid mediator related to immunity and inflammation through the activation of two types of receptors, the D-type prostanoid receptor (DP) and the chemoattractant receptorhomologous molecule expressed on Th2 cells (CRTH2), and is known to cause contractions in smooth muscle in the airway via DP and to mediate the chemotaxis of eosinophils and basophils into the lung via CRTH2 (Matsuoka et al., 2000; Hirai et al., 2001). Haematopoietic PGD synthase (H-PGDS) is known to be expressed in mast cells, antigen-presenting cells and Th2 lymphocytes (Lewis et al., 1982; Tanaka et al., 2000) and produces $\mathrm{PGD}_{2}$ from cyclooxygenase-derived $\mathrm{PGH}_{2}$, a common precursor of various PGs (Kanaoka \& Urade, 2003). Nonsteroidal anti-inflammatory drugs such as indomethacin and aspirin, both of which inhibit cyclooxygenase, suppress immune and inflammatory reactions by decreasing the production of all types of prostanoids, including cytoprotective and anti-inflammatory PGs, and induce adverse effects (Takeuchi et al., 2001; Halter et al., 2001). Therefore, H-PGDS-specific inhibitors are thought to be more useful lead compounds for anti-allergic and anti-inflammatory drugs, especially for the treatment of asthma, by decreasing the signals of $\mathrm{PGD}_{2}$ mediated by both DP and CRTH2. Aritake et al. (2006) reported the three-dimensional structure of human H-PGDS in complex with an orally active H-PGDS-specific inhibitor, 4-benzhydryloxy-1-[3-(1Htetrazol-5-yl)-propyl]-piperidine (HQL-79), which acts as an antiallergic drug, and elucidated the binding mode of HQL-79 within the catalytic pocket of H-PGDS. To develop more effective compounds, we have designed several novel H-PGDS inhibitors using HQL-79 as a lead compound and determined their binding modes in the enzymeinhibitor complexes through high-resolution X-ray crystallographic analyses. During a series of high-quality protein crystallization experiments on the International Space Station (ISS) from 2003 to 2008 which were funded by the Japan Aerospace Exploration Agency (JAXA), we focused on high-resolution crystallization and obtained high-quality crystals of H-PGDS. In this report, we report the purification and microgravity crystallization and provide a summary of the preliminary X-ray diffraction analysis of complexes of H-PGDS with three novel inhibitors for use in designing drugs based on its three-dimensional structure. 


\section{Materials and methods}

\subsection{Protein expression and purification}

Human H-PGDS was expressed recombinantly in Escherichia coli and purified as described previously (Aritake et al., 2006). In brief, E. coli BL21 (DE3) cells were transformed with the prepared plasmid carrying the cDNA of human H-PGDS. After the cells had been harvested, disrupted in phosphate-buffered saline by sonication and centrifuged, the resultant soluble fraction was applied onto a glutathione-Sepharose 4B column (GE Healthcare). H-PGDS bound to the resin was eluted with $50 \mathrm{~m} M$ Tris- $\mathrm{HCl}$ pH 9.0 containing $10 \mathrm{~m} M$ glutathione. As the preparation showed a broad band on native PAGE analysis, we further purified H-PGDS by Mono-Q HR5/5 chromatography (GE Healthcare) with a sodium chloride gradient from 0.1 to $0.2 M$ in $20 \mathrm{~m} M$ Tris- $\mathrm{HCl}$ at $293 \mathrm{~K}$. H-PGDS eluted at around $0.15 M$ sodium chloride and separated into three peaks. The fractions from the first peak were used for crystallization. The final purified sample of H-PGDS showed a single band on SDS-PAGE under reducing conditions and native PAGE under nonreducing conditions. H-PGDS was concentrated to $3.0 \mathrm{mg} \mathrm{ml}^{-1}$ in $50 \mathrm{mM}$ Tris$\mathrm{HCl}$ pH 7.5 using a Centricon YM-10 membrane (10 000 nominal molecular-weight limit; Millipore) and stored at $277 \mathrm{~K}$. The protein concentration was determined spectrophotometrically at $280 \mathrm{~nm}$.

\subsection{Inhibitors}

HQL-79 was obtained from Cayman. Three H-PGDS inhibitors, compounds $A, B$ and $C$, were synthesized at the Osaka Bioscience Institute. The formulae of the inhibitors are proprietary information. The $\mathrm{IC}_{50}$ values of the inhibitors were measured to be 400,50 and

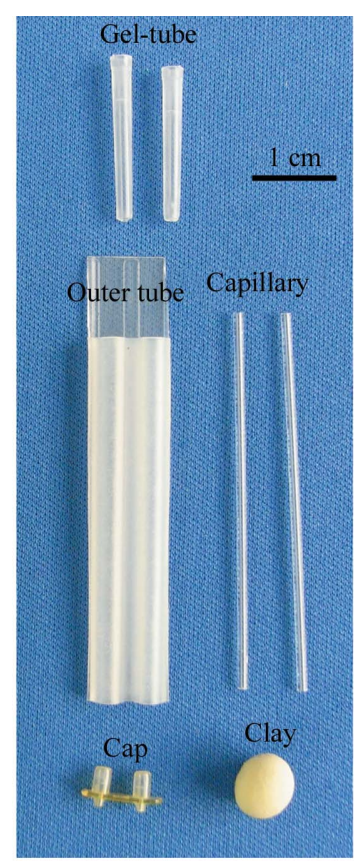

(a)

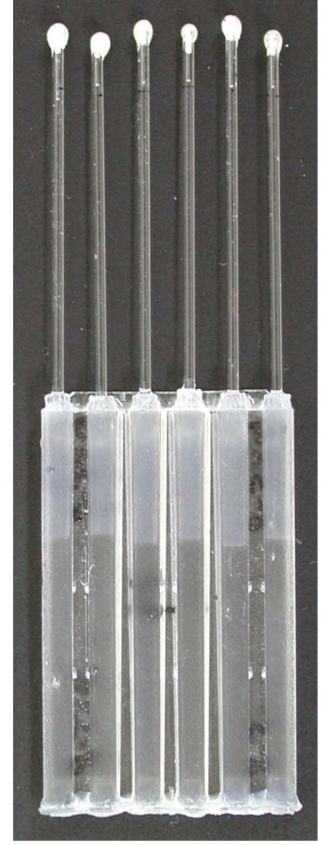

(b)

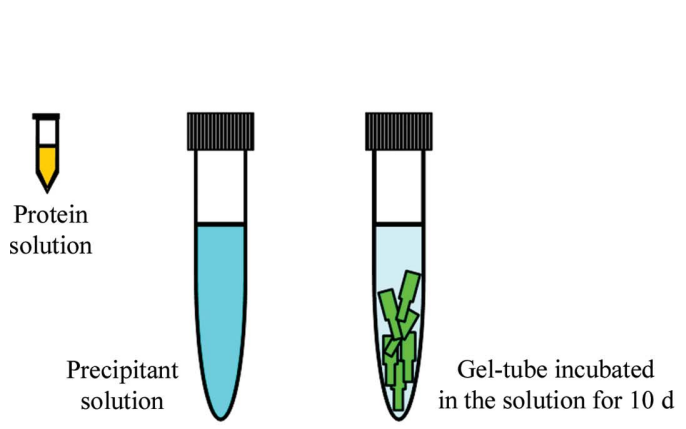

(i)

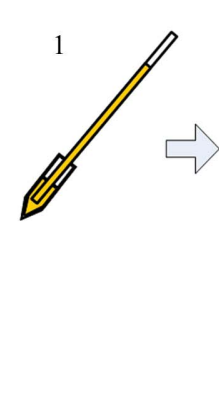

(1)

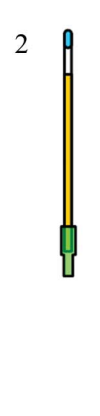

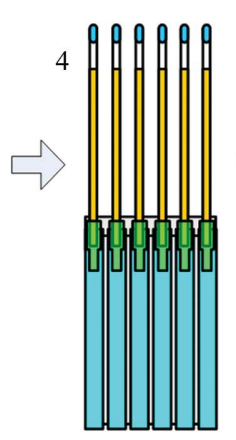

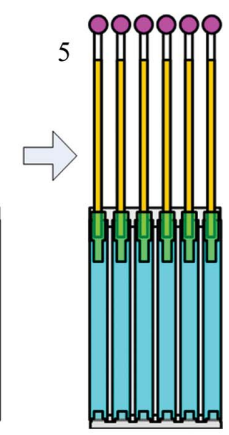

Figure 1

(ii)

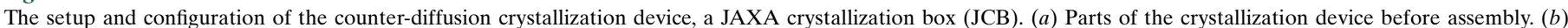

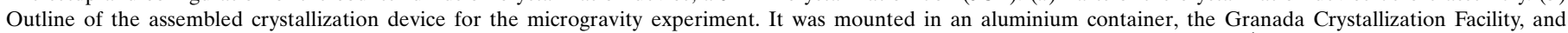

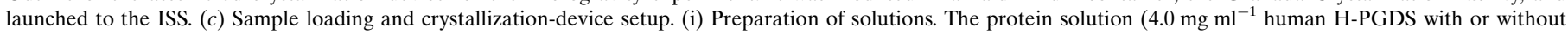

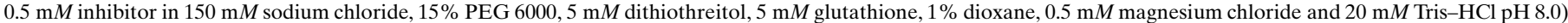

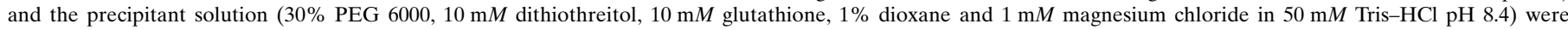

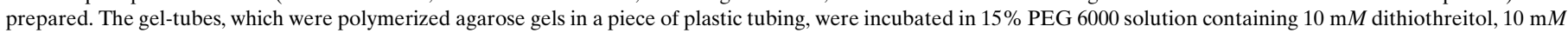

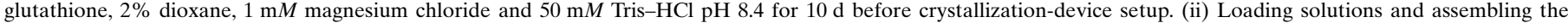

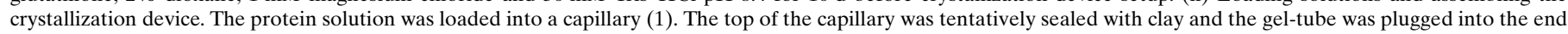

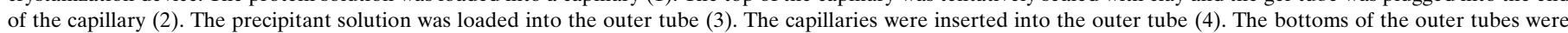
covered with caps and the top of the capillaries were completely sealed with epoxy adhesive (5). 
Table 1

Summary of X-ray diffraction experiments on H-PGDS crystals.

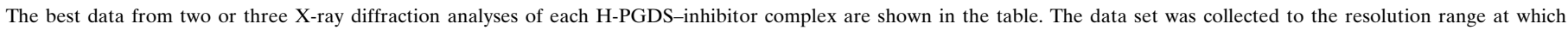
$I / \sigma(I)>2$ and $R_{\text {merge }}<50 \%$.

\begin{tabular}{|c|c|c|c|c|c|c|c|c|c|c|c|c|c|c|c|}
\hline \multirow[b]{2}{*}{ Inhibitor } & \multirow[b]{2}{*}{$\begin{array}{l}\mathrm{IC}_{50} \\
(\mathrm{n} M)\end{array}$} & \multirow[b]{2}{*}{$\begin{array}{l}\text { Space or } \\
\text { ground }\end{array}$} & \multirow[b]{2}{*}{$\begin{array}{l}\text { No. of } \\
\text { capillaries }\end{array}$} & \multirow[b]{2}{*}{$\begin{array}{l}\text { Crystal dimensions } \\
(\mathrm{mm})\end{array}$} & \multirow[b]{2}{*}{$\begin{array}{l}\text { Position in the } \\
\text { capillary } \dagger(\mathrm{mm})\end{array}$} & \multirow[b]{2}{*}{$\begin{array}{l}\text { Space } \\
\text { group }\end{array}$} & \multicolumn{6}{|c|}{ Unit-cell parameters } & \multirow[b]{2}{*}{$\begin{array}{l}\text { Mosaicity } \\
\left({ }^{\circ}\right)\end{array}$} & \multicolumn{2}{|c|}{ Maximum resolution $(\AA)$} \\
\hline & & & & & & & $(\AA)$ & $\begin{array}{l}b \\
(\AA)\end{array}$ & $\begin{array}{l}c \\
(\AA)\end{array}$ & $\begin{array}{l}\alpha \\
\left({ }^{\circ}\right)\end{array}$ & $\begin{array}{l}\beta \\
\left({ }^{\circ}\right)\end{array}$ & $\begin{array}{l}\gamma \\
\left({ }^{\circ}\right)\end{array}$ & & Data set & Observed \\
\hline \multirow[t]{2}{*}{ Free } & - & Space & 5 & $0.3 \times 0.03 \times 0.03$ & $20-30$ & $P 2_{1}$ & 48.5 & 47.3 & 184.3 & 90.0 & 97.0 & 90.0 & 0.54 & 1.5 & 1.5 \\
\hline & & Ground & 5 & $0.3 \times 0.02 \times 0.02$ & $30-40$ & $P 2_{1}$ & 48.4 & 47.4 & 184.8 & 90.0 & 97.9 & 90.0 & 2.47 & 1.7 & $末$ \\
\hline \multirow[t]{2}{*}{$A$} & 400 & Space & 3 & $0.3 \times 0.1 \times 0.05$ & $30-40$ & $P 1$ & 47.0 & 48.6 & 89.6 & 96.2 & 90.0 & 90.0 & 0.56 & 1.1 & 1.1 \\
\hline & & Ground & 3 & $0.3 \times 0.02 \times 0.02$ & $30-40$ & $P 1$ & 48.8 & 47.2 & 93.4 & 87.1 & 80.1 & 90.1 & 3.39 & 2.0 & $末$ \\
\hline \multirow[t]{2}{*}{$B$} & 50 & Space & 3 & $0.1 \times 0.1 \times 0.05$ & $30-40$ & $P 1$ & 46.9 & 48.2 & 89.4 & 83.9 & 90.0 & 90.0 & 0.62 & 1.1 & 1.1 \\
\hline & & Ground & 3 & $0.1 \times 0.1 \times 0.05$ & $30-40$ & $P 1$ & 47.1 & 48.1 & 89.7 & 84.3 & 89.9 & 89.9 & 0.81 & 1.5 & 1.8 \\
\hline \multirow[t]{2}{*}{ C } & 4400 & Space & 2 & $0.1 \times 0.1 \times 0.05$ & $30-40$ & $P 1$ & 46.9 & 48.0 & 88.9 & 84.3 & 89.9 & 89.9 & 1.48 & 1.4 & 1.8 \\
\hline & & Ground & 2 & $0.1 \times 0.1 \times 0.05$ & $30-40$ & $\S$ & $\S$ & $\S$ & $\S$ & $\S$ & $\S$ & $\S$ & $\S$ & 2.0 & $\S$ \\
\hline \multirow[t]{2}{*}{ HQL-79 } & 5900 & Space & 2 & $0.3 \times 0.1 \times 0.05$ & $30-40$ & $P 1$ & 46.9 & 48.7 & 89.3 & 95.8 & 89.9 & 90.1 & 1.71 & 1.3 & 1.3 \\
\hline & & Ground & 4 & $0.1 \times 0.1 \times 0.05$ & $30-40$ & $P 1$ & 47.0 & 48.3 & 89.2 & 95.7 & 90.2 & 89.9 & 1.28 & 1.5 & 1.5 \\
\hline
\end{tabular}

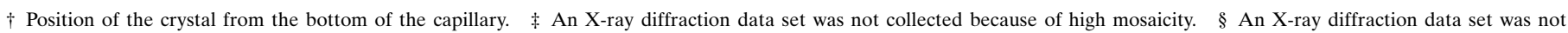
collected because of the poor quality of the diffraction.

$4400 \mathrm{n} M$, respectively, as described previously (Aritake et al., 2006). The inhibitory effects of these inhibitors were stronger than that of HQL-79 $\left(\mathrm{IC}_{50}=5900 \mathrm{n} M\right.$; Aritake et al., 2006).

\subsection{Crystallization}

H-PGDS was crystallized in a microgravity environment at $293 \mathrm{~K}$ inside a Thermal Electric Biological Universal incubator onboard the Russian Service Module on the ISS using JAXA's microgravity crystallization experiments JAXA-NGCF\#1 and JAXA-NGCF\#2 for 12 weeks from January to April 2007 and for 11 weeks from August to October 2007, respectively.

For the JAXA microgravity experiments, we adopted the JAXA Crystallization Box (JCB) as a crystallization device (Tanaka et al., $2004 a$ ), which is a modification of the original capillary counterdiffusion method (García-Ruiz \& Moreno, 1994; García-Ruiz, 2003; Ng et al., 2003; Gonzalez-Ramirez et al., 2008; Otálora et al., 2009). The set-up configuration of the JCB is shown in Fig. 1. The crystallization condition was fixed to start after the samples had been placed in the microgravity environment (about $10 \mathrm{~d}$ after sample loading) by adjusting the precipitant concentration and the gel-tube length. A microseeding technique was applied as follows: small crystals of H-PGDS were crushed in $5 \mu$ twofold-diluted precipitant solution and diluted 100 -fold with the precipitant solution; $1 \mu \mathrm{l}$ of the microseed solution was then added to $11 \mu$ protein solution. The number of capillaries for each H-PGDS inhibitor is shown in Table 1. The same crystallization condition was applied in the terrestrial control experiment.

\subsection{Data collection}

Diffraction data were collected at $100 \mathrm{~K}$ with an X-ray wavelength of $0.85 \AA$ on the BL41XU beamline at SPring-8, Harima, Japan using an ADSC315 detector system. The crystals grown in the capillaries were extracted into artificial mother liquor (30\% PEG 6000, $10 \mathrm{mM}$ dithiothreitol, $10 \mathrm{~m} M$ glutathione, $1 \%$ dioxane and $1 \mathrm{~m} M$ magnesium chloride in $50 \mathrm{~m} M$ Tris- $\mathrm{HCl} \mathrm{pH}$ 8.4). The concentration of PEG 6000 in the artificial mother liquor was calculated using a one-dimensional simulation program that estimates the time-course of the concentration change of the precipitant solution at a certain position in the capillary (Tanaka et al., 2004a). The method of harvesting crystals has been reported previously (Tanaka et al., 2007). One crystal was selected with a nylon loop, briefly dipped into artificial mother liquor supplemented with $15 \%$ glycerol as a cryoprotectant for less than $10 \mathrm{~s}$ and plunged into a nitrogen-gas stream at $100 \mathrm{~K}$. A total of 180 frames were collected using a crystal-to-detector distance of $150 \mathrm{~mm}$ with $1^{\circ}$ oscillation. The diffraction images were integrated and scaled using the programs DENZO and SCALEPACK from the $H K L-2000$ suite (Otwinowski \& Minor, 1997). We performed X-ray diffraction experiments on two or three crystals and summarize the best data for each complex in Table 1. Data sets were collected to the resolution range where $I / \sigma(I)>2$ and $R_{\text {merge }}<50 \%$.

\section{Results and discussion}

\subsection{Visual inspection of crystals}

Two or three crystals grew in every $10 \mathrm{~mm}$ of the capillaries both on terra firma and in microgravity. The dimensions of the crystals grown terrestrially and in microgravity were almost the same $(0.3 \times$ $0.1 \times 0.05 \mathrm{~mm})$. However, the crystals grown in microgravity did not form clusters (Fig. 2), suggesting that the crystal quality was improved by crystallization in microgravity. The number and size of the crystals did not change in the presence or the absence of H-PGDS inhibitors.

\subsection{X-ray diffraction}

The results of the X-ray diffraction are summarized in Table 1. All crystals chosen for diffraction data collection were of sufficient size and of good quality as judged by visual inspection. In the counterdiffusion method, crystals of better quality tend to grow at the opposite side to the gel-tube, in the section of the capillary where the concentration change of the precipitant is slower (Lopez-Jaramillo et al., 2003). As a result, the crystals grown in capillaries at $30-40 \mathrm{~mm}$ from the gel-tube of the capillary were used for X-ray diffraction experiments in most of the cases. To avoid fluctuations in the data caused by crystal size differences, we chose microgravity-grown and terrestrial-grown crystals of the same size for X-ray diffraction experiments. Crystals of different space groups $(P 1)$ were obtained in the presence of inhibitors, owing to the binding of the inhibitor, compared with those grown in the absence of inhibitors $\left(P 2_{1}\right)$.

Terrestrial-grown crystals of H-PGDS in the presence of inhibitor $B$ and HQL-79 exhibited X-ray data sets to 1.8 and $1.5 \AA$ resolution with mosaicities of 0.81 and 1.28 , respectively (Table 1 ). Although the H-PGDS crystals grown in the absence of inhibitor (space group $P 2_{1}$ ) and in the presence of inhibitors $A$ and $C$ showed X-ray diffraction to 1.7, 2.0 and $2.0 \AA$ resolution, respectively, we did not collect their $\mathrm{X}$-ray data sets because of relatively high mosaicity or poor-quality 
diffraction. In contrast, microgravity-grown crystals in the absence or the presence of inhibitors $A, B, C$ and HQL-79 exhibited X-ray data sets to $1.5,1.1,1.1,1.8$ and $1.3 \AA$ resolution with mosaicities of 0.54 , $0.56,0.62,1.48$ and 1.71 , respectively (Table 1 ).

\subsection{Affinity of inhibitors for H-PGDS}

Among the microgravity-grown crystals, both the maximum resolution and the mosaicity of the X-ray diffraction data are relatively low in the complexes with inhibitors with low $\mathrm{IC}_{50}$ values (Table 1). This may be a consequence of immobilization of the catalytic pocket of H-PGDS after binding the high-affinity inhibitors in the enzymeinhibitor complexes, leading to the growth of well ordered crystals in microgravity.

Recently, novel inhibitors of H-PGDS have been generated by fragment-based drug design and are expected to contribute to novel drug development (Hohwy et al., 2008). The best inhibitor showed an $\mathrm{IC}_{50}$ of $21 \mathrm{n} M$ and is one of the most potent inhibitors described to date. Such high-affinity inhibitors may also improve the crystal quality of H-PGDS-inhibitor complexes in the microgravity environment, as shown in this report.

\subsection{The counter-diffusion method in microgravity}

The counter-diffusion method used in this report is based on the counter-diffusion of protein and precipitant along a capillary. Using this method in a convection-free environment such as microgravity or gels, uniform propagation of the supersaturation wave is expected in the capillary, which enables simultaneous screening for optimal conditions for protein crystallization. Moreover, in a convection-free environment a protein-depletion zone (PDZ) and an impuritydepletion zone (IDZ) are formed around the growing crystal, which are believed to be beneficial for improving crystal quality (McPherson, 1999; Chernov, 1998; Thomas et al., 2000). In addition,

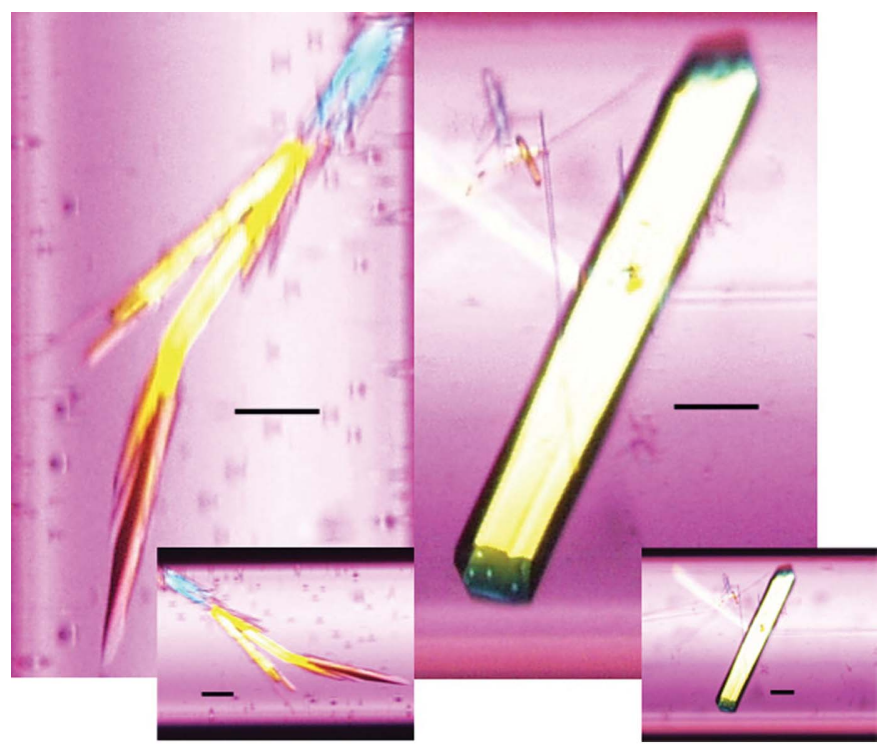

(a)

(b)

Figure 2

Crystals of H-PGDS grown under terrestrial $(a)$ and microgravity $(b)$ conditions. In the terrestrial experiment the size of the crystal was almost the same for all of the complexes. Crystals were first observed at around $10 \mathrm{~mm}$ from the bottom of the capillary after two weeks of crystallization setup and were observed at the top of the capillary ( $40 \mathrm{~mm}$ from the bottom) after 12 weeks of crystallization setup. The crystals had a tendency to form clusters. In the microgravity experiment plate-like crystals were grown with good morphology. The scale bars correspond to $100 \mu \mathrm{m}$. these zones are formed much more significantly in a highly viscous solution such as PEG (Tanaka et al., 2004b) and with highly purified protein (Yamanaka et al., 2009). Since the numbers of crystals that we have used here were very limited, we avoid any comparative statements; however, these might have positive effects on the growth of high-quality crystals in microgravity in our cases.

\section{Conclusions}

We obtained high-quality crystals of H-PGDS complexes with inhibitors by the counter-diffusion method in a microgravity environment. The counter-diffusion method in microgravity is the only way to obtain crystals of H-PGDS inhibitor complexes that diffract to 1.0$1.5 \AA$ resolution. This will provide us with a better understanding of the binding mode of H-PGDS with inhibitors for future drug design. Structural refinement is currently in progress.

It is still difficult to identify any predictive technique to determine whether a crystallization experiment will benefit from microgravity; however, many examples of improved crystal quality in microgravity have been reported (Littke \& John, 1986; DeLucas et al., 1989; Day \& McPherson, 1992; He \& Carter, 1992; Borgstahl et al., 2001; Kundrot et al., 2001; Sauter et al., 2001; Vergara et al., 2003; Snell \& Helliwell, 2005; Evrard et al., 2007; Tanaka et al., 2007; Meyer et al., 2008). The complexes of human H-PGDS with high-affinity inhibitors are new examples of improved crystal quality in microgravity.

This work was supported by the Program for Promotion of Fundamental Studies in Health Sciences of the National Institute of Biomedical Innovation (NIBIO). This study was funded in part through the High-Quality Protein Crystal Growth Experiment on JEM promoted by JAXA. We are grateful to the Federal Space Agency and RSC Energia for the use of the Russian Service Module and the Russian spacecrafts Progress and Soyuz for space transportation, to ESA (European Space Agency) and the University of Granada for space crystallization technology and to the Japan Synchrotron Radiation Research Institute (JASRI) for access to and user support at the synchrotron facilities of BL41XU at SPring-8, Harima, Japan.

\section{References}

Aritake, K., Kado, Y., Inoue, T., Miyano, M. \& Urade, Y. (2006). J. Biol. Chem. 281, 15277-15286.

Borgstahl, G. E. O., Vahedi-Faridi, A., Lovelace, J., Bellamy, H. D. \& Snell, E. H. (2001). Acta Cryst. D57, 1204-1207.

Chernov, A. A. (1998). Acta Cryst. A54, 859-872.

Day, J. \& McPherson, A. (1992). Protein Sci. 1, 1254-1268.

DeLucas, L. J. et al. (1989). Science, 246, 651-654.

Evrard, C., Maes, D., Zegers, I., Declercq, J.-P., Vanhee, C., Martial, J., Wyns, L. \& Van De Weerdt, C. (2007). Cryst. Growth Des. 7, 2161-2166.

García-Ruiz, J. M. (2003). Methods Enzymol. 368, 130-154.

García-Ruiz, J. M. \& Moreno, A. (1994). Acta Cryst. D50, 484-490.

Gonzalez-Ramirez, L. A., Carrera, J., Gavira, J. A., Melero-Garcia, E. \& García-Ruiz, J. M. (2008). Cryst. Growth Des. 8, 4324-4329.

Halter, F., Tarnawski, A. S., Schmassmann, A. \& Peskar, B. M. (2001). Gut, 49, $443-453$.

He, X. M. \& Carter, D. C. (1992). Nature (London), 358, 209-215.

Hirai, H., Tanaka, K., Yoshie, O., Ogawa, K., Kenmotsu, K., Takamori, Y., Ichimasa, M., Sugamura, K., Nakamura, M., Takao, S. \& Nagata, K. (2001). J. Exp. Med. 193, 255-261.

Hohwy, M., Spadola, L., Lundquist, B., Hawtin, P., Dahmen, J., Groth-Clausen, I., Nilsson, E., Persdotter, S., von Wachenfeldt, K., Folmer, R. H. A. \& Edman, K. (2008). J. Med. Chem. 51, 2178-2186.

Kanaoka, Y. \& Urade, Y. (2003). Prostaglandins Leukot. Essent. Fatty Acids, 69, 163-167. 


\section{crystallization communications}

Kundrot, C. E., Judge, R. A., Pusey, M. L. \& Snell, E. H. (2001). Cryst. Growth Des. 1, 87-99.

Lewis, R. A., Soter, N. A., Diamond, P. T., Austen, K. F., Oates, J. A. \& Roberts, L. J. II (1982). J. Immunol. 129, 1627-1631.

Littke, W. \& John, C. (1986). J. Cryst. Growth, 76, 663-672.

Lopez-Jaramillo, F. J., Otálora, F. \& Gavira, J. A. (2003). J. Cryst. Growth, 247, 177-184.

Matsuoka, T. et al. (2000). Science, 287, 2013-2017.

McPherson, A. (1999). Crystallization of Biological Macromolecules. Cold Spring Harbor Laboratory Press.

Meyer, A., Rypniewski, W., Szymański, M., Voelter, W., Barciszewski, J. \& Betzel, C. (2008). Biochim. Biophys. Acta, 1784, 1590-1595.

Ng, J. D., Gavira, J. A. \& García-Ruiz, J. M. (2003). J. Struct. Biol. 142, 218 231.

Otálora, F., Gavira, J. A., Ng, J. D. \& García-Ruiz, J. M. (2009). Prog. Biophys. Mol. Biol. 101, 26-37.

Otwinowski, Z. \& Minor, W. (1997). Methods Enzymol. 276, 307326.
Sauter, C., Otálora, F., Gavira, J.-A., Vidal, O., Giegé, R. \& García-Ruiz, J. M. (2001). Acta Cryst. D57, 1119-1126.

Snell, E. H. \& Helliwell, J. R. (2005). Rep. Prog. Phys. 68, 799-853.

Takeuchi, K., Araki, H., Umeda, M., Komoike, Y. \& Suzuki, K. (2001). J. Pharmacol. Exp. Ther. 297, 1160-1165.

Tanaka, H. et al. (2007). Acta Cryst. F63, 69-73.

Tanaka, H., Inaka, K., Sugiyama, S., Takahashi, S., Sano, S., Sato, M. \& Yoshitomi, S. (2004a). J. Synchrotron Rad. 11, 45-48.

Tanaka, H., Inaka, K., Sugiyama, S., Takahashi, S., Sano, S., Sato, M. \& Yoshitomi, S. (2004b). Ann. NY Acad. Sci. 1027, 10-19.

Tanaka, K., Ogawa, K., Sugamura, K., Nakamura, M., Takano, S. \& Nagata, K. (2000). J. Immunol. 164, 2277-2280.

Thomas, B. R., Chernov, A. A., Vekilov, P. G. \& Carter, D. C. (2000). J. Cryst. Growth, 211, 149-156.

Vergara, A., Lorber, B., Zagari, A. \& Giegé, R. (2003). Acta Cryst. D59, 2-15. Yamanaka, M., Tanaka, H., Takahashi, S., Inaka, K., Nakagawa, A., Sato, M., Kobayashi, T. \& Tanaka, T. (2009). The 9th Annual Meeting of the Protein Science Society of Japan Abstract Book, p. 119. 